\title{
EFFECTIVENESS OF FRAGILITY FRACTURE INTEGRATED REHABILITATION MANAGEMENT FOR IMPROVING ACTIVITY OF DAILY LIVING AND QUALITY OF LIFE IN GERIATRIC POPULATION AFTER HIP FRACTURE
}

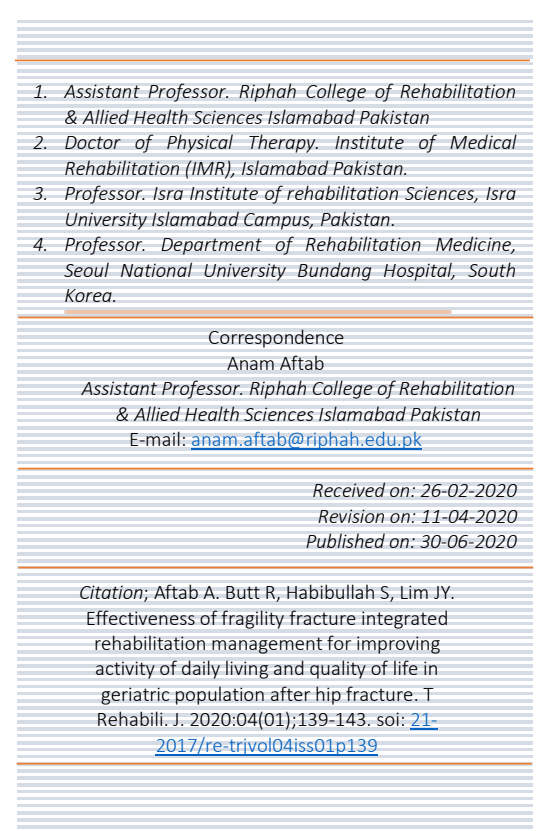

Anam Aftab $^{1}$ : Conception \& Designing, Analysis \& interpretation of data, writing; Revised and accountable for all aspects Rizwana Butt ${ }^{2}$ : Analysis \& interpretation of data, writing; Revised and Accountable for all aspects. Shaista Habibullah ${ }^{3}:$ Conception \& Designing, Revised and accountable for all aspects

Jae Young Lim ${ }^{4}$ : Analysis \& interpretation of data, writing; Revised and Accountable for all aspects ABSTRACT

Objective: The objective of the study was to determine the efficacy of Fragility Integrated Rehabilitation Management (FIRM) on the quality of life and activities of daily living in geriatric population with hip fracture and to reduce the recurrence of hip fracture. Material and Method: A single-group, pretest-posttest design was conducted at Seoul National University for a time period from August 2017 to January 2018 in Bundang Hospital, South Korea. Patients with age of 55 years and above, had diagnosed femoral neck fractures, reduction and internal fixation, intertrochanteric and sub-trochanteric fractures, total hip replacement (THR), bipolar hemi-arthroplasty were included in the study. Quality of life was assessed through the Euro-QOL (EQ-5D) questionnaire, while ADL's was measured through Modified Barthal Index (MBI). Non-parametric Wilcoxon Sign Rank test was used for prepost analysis by using SPSS version 21 . The level of significance was set at $95 \% \mathrm{Cl}(p<0.05)$. Results: Wilcoxon signed rank test showed a significant improvement in activities of daily living $(p<0.05)$. Furthermore, significant improvement has also been determined in the quality of life $(p<0.05)$, after the $10^{\text {th }}$ Fragility Integrated Rehabilitation Management (FIRM) and 4 Occupational Therapy (OT) sessions. Conclusion: Fragility Integrated Rehabilitation Management showed a significant improvement in the Activities of Daily Living (ADLs) and Quality of Life (QOL).

Keywords: Fragility fracture, geriatric, hip fracture, physical therapy, occupational therapy, rehabilitation.

\section{INTRODUCTION}

Osteoporosis is a common factor which causes most of the fragility fractures in older population which causes severe problems including daily activity limitations and gait impairments. ${ }^{1-3}$ Vertebra, humerus, radius, hip are the most common sites. ${ }^{1,2}$ The incidence of fracture and disability after fracture increases with time due to the demographic changes and increase in life expectancy. $^{3}$

In 2018, South Korea became an aged society, $14.3 \%$ population consists on adults' aged $\geq 65$ years, and by 2025, it can become a super-aged society. ${ }^{3}$ Moreover in South Korea, from 2008 to 2012, the incidence of hip fracture increased by 14.1\%. And after the hip fracture, mortality rate during the first year is $8.4-36 \%$. Mortality rate is higher in hip fracture as compared to non-hip fracture and control population. ${ }^{4}$

Fragility hip fracture is a geriatric condition with comorbidities $^{5,6}$ which increase socioeconomic burden because of the increased expenses of medical care. In spite of the fact that the treatment quality of hip fracture has been improved but there are still lacking of the functional recovery of the patient after surgery, thus it is a need to provide comprehensive and multidisciplinary rehabilitation to restore the functional recovery after the surgery. ${ }^{5,7}$ In a previous study conducted by Abraham Adunsky et al. described the effectiveness and importance of comprehensive rehabilitation care for geriatric population after fragility hip fracture. $^{8}$

For the purpose of comprehensive rehabilitation care, a multidisciplinary hip fracture care program comprising of orthopedic doctors, physiotherapists, occupational therapists, nurses geriatricians was designed, which is known as Fracture Integrated Rehab Management (FIRM). The main goal of FIRM was to increase the level of independence, prevention of falls and detailed discharge planning. It consist on 10 days of physiotherapy and 4 days for occupational therapy sessions after surgery, increasing gradually depending on patients' functional level. ${ }^{7}$

In this study a new rehabilitation strategy, FIRM which was ascertained by Korean Fragility Fracture Rehabilitation Study group ${ }^{7}$, was used. If the research hypothesis is proved, then it would be a big step in rehabilitation for the cases with Fragility fractures, which are rising day by day due to advancing age. ${ }^{9,10}$ The aim of the study was to determine the efficacy of Fragility Integrated 
Rehabilitation Management (FIRM) on the quality of life and activities of daily living in geriatric population with hip fracture and to reduce the recurrence of hip fracture. This system will contribute public health by increasing quality of life in older population with fragility fracture and improve their functional recovery.

\section{METHODOLOGY}

A single-group, pretest-posttest design was conducted at Seoul National University for a time period from August 2017 to January 2018 in Bundang Hospital, South Korea. Patients with age of 55 years and above, had diagnosed femoral neck fractures, reduction and internal fixation, intertrochanteric and sub-trochanteric fractures, total hip replacement (THR), bipolar hemiarthroplasty were included in the study. Subjects were excluded if the patients had multiple fractures, revised hip surgeries, isolated greater and lesser tuberosity fractures, fractures due to infection and malignancy, or had a medical condition due to which rehabilitation wasn't possible, and declined to participate in the clinical trials. The study was started after approval from the research committee of Institutional Review Board of Seoul National University Bundang Hospital. Informed consent according to Declaration of Helsinki, was taken from each subject and assured them about the confidentiality of their data.

A total of $n=14$ patients fulfilled the eligibility criteria and received the FIRM protocol, which is a multidisciplinary team approach, and comprehensive rehabilitation program. FIRM was provided by rehabilitation physician, clinical nurse specialist, nutritionist, physical therapist, occupational therapist and a social worker. Participants had been provided by 2 weeks long intervention after surgery, which contained 10 days physiotherapy sessions and 4 days of occupational therapy. Each session lasts for 40mins/day (20 minutes twice a day) as shown in table 1.

The pre-interventional data was collected on $2^{\text {nd }}$ postoperative day and post-interventional on $15^{\text {th }}$ postoperative after the $10^{\text {th }}$ FIRM session. The general demographic data in term of age, gender and BMI was obtained at the baseline. Quality of life or health status of the participants was assessed through the Euro-QOL (EQ-5D) questionnaire $^{11}$, while ADL's was measured through
Modified Barthal Index (MBI), and low score indicated a worse outcome. ${ }^{12}$ As both variable were ordinal, so non parametric Wilcoxon Sign Rank test was used for pre-post analysis by using SPSS version 21 . The level of significance was set at $95 \%$ $\mathrm{Cl}(p<0.05)$.

\section{RESULTS}

The mean age of study participant was $82.07 \pm 6.0$ and BMI was $22.71 \pm 4.08$. A total of $n=14$ participants were recruited for the study. Wilcoxon signed rank test showed a significant improvement in modified Barthal index (MBI). In $\mathrm{MBI}$, self-bathing, toilet, stair climbing, dressing, ambulation, transfer is significantly improved $(p<0.05)$. The personal hygiene, feeding, wheel chair, and bowel-bladder control didn't show any significant improvement $(p \geq 0.05)$ after $10^{\text {th }}$ Fragility Integrated Rehabilitation Management (FIRM) and 4 Occupational Therapy (OT) sessions. Furthermore, significant improvement has been determined in the quality of life. In QOL-EQ-5D, mobility, self-care, usual activities, pain/discomfort is significantly improved $(p<0.05)$. However, no significant improvement has been seen in the depression/anxiety $(p \geq 0.05)$, after the $10^{\text {th }}$ Fragility Integrated Rehabilitation Management (FIRM) and 4 Occupational Therapy (OT) sessions.

\section{DISCUSSION}

The aim of the study was to determine the efficacy of Fragility Integrated Rehabilitation Management (FIRM) on the quality of life (QOL) and activities of daily living ( $A D L$ 's) in geriatric population with hip fracture and to reduce the recurrence of hip fracture. The post-intervention analysis showed significant improvement in activities of daily living on Modified Barthal index. Previous studies demonstrated that due to decrease in rehabilitation, $^{13}$ hip fractures leads to decrease functional independence and worsens the quality of life. ${ }^{14}$ While, rehabilitation has an important role in improving quality of life and functional independence of hip fracture patients. ${ }^{15}$ The findings of current study showed that FIRM protocol significantly improved bathing, toilet, dressing, stair climbing, ambulation walking with or without aid and transfers from bed to chair or back $(p<0.05)$ after the $10^{\text {th }}$ FIRM session, which correlates with the previous studies and significant improvement has been seen in the mobility and activities of daily living. ${ }^{7,} 16$ However, in frail elder population improvements in mobility and ADL's is of greater importance to cope up with the activities of daily life. ${ }^{17}$ 
Table 1: Fragility Fracture Integrated Rehabilitation Management (FIRM) Protocol

\begin{tabular}{|c|c|c|}
\hline & Physical therapy protocol & Occupational Therapy Protocol \\
\hline 1* Session & Evaluate to check out status of the subject. & \\
\hline \multirow{4}{*}{$2^{\mathrm{d}}$ Session } & $\begin{array}{l}\text { Carry out hip ROM (AAROM and AROM) exercise according to status, level, } \\
\text { function of subject }\end{array}$ & \\
\hline & $\begin{array}{l}\text { Carry out functional training on the mat for progressive exercise } \\
\text { and stable function depending on the level of the subject (ex. Mat } \\
\text { training - rolling, sit up, scooting) }\end{array}$ & \\
\hline & $\begin{array}{l}\text { Carry out strengthening exercise (hip abductor \& extensor) \& } \\
\text { education depending on the level of the subject (on a mat). (If } \\
\text { good motivation, re-training can be possible in the ward) }\end{array}$ & \\
\hline & $\begin{array}{l}\text { Carry out cardiovascular exercise for lower cardiopulmonary function } \\
\text { due to bedding or aging depending on the level of the subject (ex. } \\
\text { Thera Vital, UBE, daily application) }\end{array}$ & \\
\hline \multirow{3}{*}{$3^{\text {d Session }}$} & $\begin{array}{l}\text { Carry out tilt table standing for confirming the orthostatic hypotension } \\
\text { of the subject's condition. }\end{array}$ & \\
\hline & $\begin{array}{l}\text { Carry out sit to stand exercise depending on the level of the } \\
\text { subject }\end{array}$ & \\
\hline & $\begin{array}{l}\text { Carry out cardiovascular exercise for lower cardiopulmonary function } \\
\text { due to bedding or aging depending on the level of the subject (ex. } \\
\text { Thera Vital, UBE, daily application) }\end{array}$ & \\
\hline \multirow{4}{*}{$4^{\text {th Sessian }}$} & $\begin{array}{l}\text { Carry out non-weight bearing exercise in P-bar depending on the } \\
\text { level of the subject (if stable, do next stage) }\end{array}$ & \multirow{4}{*}{ Initial assessment (status, level, function) } \\
\hline & $\begin{array}{l}\text { Carry out P-bar walking depending on the level of the subject(if } \\
\text { can carry outP-bar gait } 3 \text { roll, do next stage) }\end{array}$ & \\
\hline & $\begin{array}{l}\text { Carry out strengthening exercise (hip abductor \& extensor)\& } \\
\text { education depending on the level of the subject (on a mat). (If } \\
\text { good motivation, re-training can be possible in the ward) }\end{array}$ & \\
\hline & $\begin{array}{l}\text { Carry out cardiovascular exercise for lower cardiopulmonary function } \\
\text { due to bedding or aging depending on the level of the subject (ex. } \\
\text { Thera Vital, UBE, daily application) }\end{array}$ & \\
\hline \multirow{3}{*}{ 5r Session } & $\begin{array}{l}\text { Go ahead with a gait endurance of } 50 \mathrm{~m}(25 \mathrm{~m} / \mathrm{min}=0.4 \mathrm{~m} / \mathrm{s}) \text {. And } \\
\text { carry out walking depending on the level of the subject. (Proceed in } \\
\text { the order of P-bar, walker depending on the level of the subject) }\end{array}$ & \\
\hline & $\begin{array}{l}\text { Carry out gait with assist \& supervision with rolling walker (You should } \\
\text { check the gait pattern \& posture) }\end{array}$ & \\
\hline & $\begin{array}{l}\text { Carry out cardiovascular exercise for lower cardiopulmonary function } \\
\text { due to bedding or aging depending on the level of the subject (ex. } \\
\text { Thera Vital, UBE, daily application) }\end{array}$ & \\
\hline Gl Session & Same as $5^{\text {th }}$ session & $\begin{array}{l}\text { ADL taining } \\
\text { 1. Transfer: W/C to chair, W/C to bed, W/C to } \\
\text { toilet, sit to standing } \\
\text { 2. Bed mobility : sit up, roll over } \\
\text { 3. Dressing : education of wearing pants with } \\
\text { Reacher and purchasing } \\
\text { 4. Home environment improvement: installing } \\
\text { non-slip mat, safe-bar in the toilet } \\
\text { Education of precautions after hip fracture surgery } \\
\text { (Total Hip Replacement Arthroplasty, Complete Hip } \\
\text { Replacement, Hemiarthroplasty, Internal Fixation). }\end{array}$ \\
\hline Th Session & Same as $5^{\text {th }}$ session & \\
\hline \multirow{3}{*}{$8^{\text {th Session }}$} & Carry out stair ambulation depending on the level of the subject. & \multirow{3}{*}{$\begin{array}{l}\text { After confirming improved ADL training and } \\
\text { function, re-educate deficient areas }\end{array}$} \\
\hline & $\begin{array}{l}\text { Perform treadmill exercise }(1.4 \mathrm{~km} / \mathrm{h}) \text { depending on the level of } \\
\text { the subject. }\end{array}$ & \\
\hline & $\begin{array}{l}\text { Carry out cardiovascular exercise for lower cardiopulmonary function } \\
\text { due to bedding or aging depending on the level of the subject (ex. } \\
\text { Thera Vital, UBE, daily application) }\end{array}$ & \\
\hline 9h Session & Same as $8^{\text {th }}$ Session & \\
\hline $10^{h}$ Session & Evaluate to check out status of the subject. & Final assessment (status, level, function) \\
\hline
\end{tabular}

Table 2: Pre \& Post analysis of ADLs \& QOL

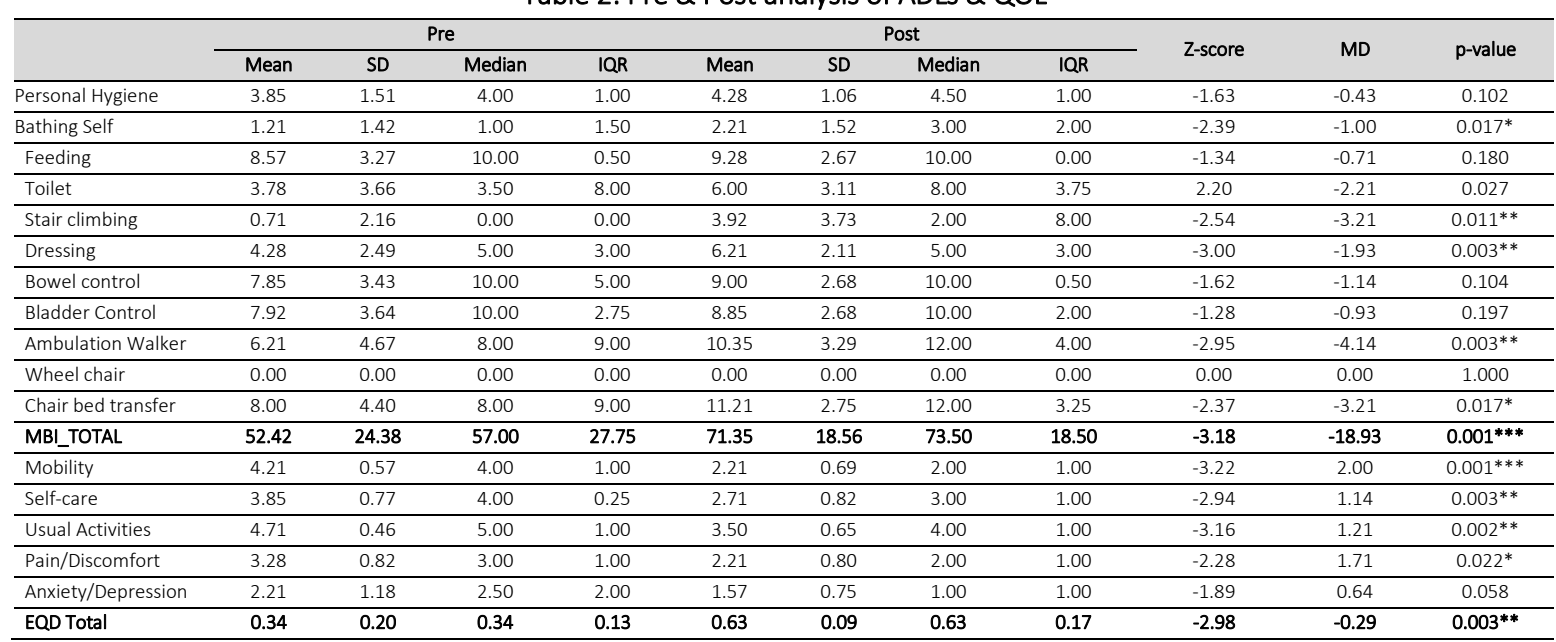

Level of Significance, $P$-value $<0.05^{*}, p$-value $<0.01^{* *}, p$-value $<0.001^{* * *}$ 
Moreover, in the recent study subjects were assessed after the 4 days of occupational therapy sessions along with Fragility Integrated Rehabilitation Management (FIRM), which showed a significant improvement in dressing, toilet and bathing. T. Alarcon et al. conducted a 2-years follow study, which correlates with the current findings and significant improvement has been seen in the activities of daily living such as ambulation, transfers, stair climbing, use of toilet, bathing and dressing $(p<0.05) .^{18}$

Furthermore, significant improvement has been determined in mobility, self-care, usual activities and pain/discomfort after the $10^{\text {th }}$ Fragility Integrated Rehabilitation Management (FIRM) and $4^{\text {th }}$ Occupational Therapy (OT) session. In a study conducted by Shyu Yl et al., it was determined the interdisciplinary team approach is effective for management of hip fracture in geriatric population and improve their quality of life by decreasing pain and improve physical function. ${ }^{19}$ Moreover, previous literature determined that the Fragility Integrated Rehabilitation Management (FIRM) can significantly improve the quality, joint range of motion and decrease in pain/discomfort in elder population with hip fracture which supports the findings of current study. ${ }^{20}$

FIRM protocol included comprehensive management which was provided by multidisciplinary team such as physical therapist, occupational therapist, clinical nurse, nutritionist and a social worker, which could be an important part for the management of the hip fracture in elderly population. ${ }^{21}$ In some researches, it has been concluded that comprehensive management is effective in improving functional independence and activities of daily life as compared to the conventional physiotherapy. ${ }^{22}$

In this study, anxiety and depression is not significantly improved $(p \geq 0.05)$. While, depression could be a factor to decrease the mobility and functional outcomes in elderly population with hip fracture. ${ }^{23}$ But a study reported, 1 year-long followup determined a decrease in depression after discharge from the hospital. ${ }^{19}$

The limitation of this study is that it was single group, one centred study. Moreover, sample size was also small, so results can't be generalized.

\section{CONCLUSION}

FIRM showed an improvement in the mobility, selfcare activities, ambulation, stair climbing, and transfer. Another improvement has been also observed in the decrease in pain and discomfort due to the Hip fracture. Furthermore, activities of daily living are significantly improved, which demonstrated that FIRM is an effective protocol for hip fracture in geriatric population. It is suggested that in future studies, such conditions such as metabolic diseases and metabolic bone diseases which may hinders the improvement in hip fracture should be considered. Large sample size and multicenter studies should be incorporated in future researches.

\section{REFERENCES}

1. Lems WF, Dreinhöfer $K$, Bischoff-Ferrari $H$, Blauth $M$, Czerwinski $E$, da Silva JA, et al. EULAR/EFORT recommendations for management of patients older than 50 years with a fragility fracture and prevention of subsequent fractures. Ann. Rheum. Dis 2017;76(5):802-10.

2. Riggs BL, Melton lii $\mathrm{L}$. The worldwide problem of osteoporosis: insights afforded by epidemiology. B. 1995;17(5):S505-S11.

3. Lim S-K, Lee SY, Beom J, Lim J-Y. Comparative outcomes of inpatient fragility fracture intensive rehabilitation management (FIRM) after hip fracture in sarcopenic and non-sarcopenic patients: a prospective observational study. Eur. Geriatr. Med. 2018;9(5):641-50.

4. Abrahamsen B, Van Staa T, Ariely R, Olson M, Cooper C. Excess mortality following hip fracture: a systematic epidemiological review. Osteoporos. Int. 2009;20(10):1633-50.

5. Lim J-Y. Fragility Fracture Care: An Urgent Need to Implement the Integrated Model of Geriatric Care. Ann. Geriatr. Med. Res.. 2019;23(1):1-2.

6. Menzies IB, Mendelson DA, Kates SL, Friedman SM. The impact of comorbidity on perioperative outcomes of hip fractures in a geriatric fracture model. Geriatr. Orthop. Surg. Rehabil.. 2012;3(3):129-34.

7. Lee SY, Beom J, Kim BR, Lim S-K, Lim J-Y. Comparative effectiveness of fragility fracture integrated rehabilitation management for elderly individuals after hip fracture surgery: a study protocol for a multicenter randomized controlled trial. Med. 2018;97(20).

8. Adunsky A, Lerner-Geva L, Blumstein T, Boyko V, Mizrahi E, Arad M. Improved survival of hip fracture patients treated within a comprehensive geriatric hip fracture unit, compared with standard of care treatment. J. Am. Med. Dir. Assoc. 2011;12(6):439-44.

9. Curtis EM, Moon RJ, Harvey NC, Cooper C. Reprint of: The impact of fragility fracture and approaches to osteoporosis risk assessment worldwide. Int. J. Orthop. Trauma. Nurs. 2017;26:7-17.

10. Barnes B, Dunovan K. Functional outcomes after hip fracture. Phys. Ther. 1987;67(11):1675-9.

11. Obradovic $M$, Lal $A$, Liedgens $H$. Validity and responsiveness of EuroQol-5 dimension (EQ-5D) versus Short Form-6 dimension (SF-6D) questionnaire in chronic pain. Health. Qual. Life. Outcomes. 2013;11(1):110.

12. Jung HY, Park BK, Shin HS, Kang YK, Pyun SB, Paik NJ, et al. Development of the Korean Version of Modified Barthel 
Index (K-MBI): Multi-center Study for Subjects with Stroke. Ann. Rehabil. Med. 2007;31(3):283-97.

13. Young $\mathrm{Y}$, Xiong $\mathrm{K}$, Pruzek RM. Longitudinal functional recovery after postacute rehabilitation in older hip fracture patients: the role of cognitive impairment and implications for long-term care. J. Am. Med. Dir. Assoc. 2011;12(6):431-8.

14. Randell A, Nguyen $T$, Bhalerao $N$, Silverman $S$, Sambrook $P$, Eisman J. Deterioration in quality of life following hip fracture: a prospective study. Osteoporos. Int. 2000;11(5):460-6.

15. Abdalbary SA. Partial weight bearing in hip fracture rehabilitation. Future Sci.; 2017.

16. Aftab A, Awan WA, Habibullah S, Lim JY. Effects of fragility fracture integrated rehabilitation management on mobility, activity of daily living and cognitive functioning in elderly with hip fracture. PAK. J. MED. SCI. 2020;36(5).

17. Nordström P, Thorngren K-G, Hommel A, Ziden L, Anttila S. Effects of geriatric team rehabilitation after hip fracture: meta-analysis of randomized controlled trials. J. Am. Med. Dir. Assoc. 2018;19(10):840-5.

18. Alarcón T, González-Montalvo J, Gotor P, Madero R, Otero A. Activities of daily living after hip fracture: profile and rate of recovery during 2 years of follow-up. Osteoporos. Int. 2011;22(5):1609-13.

19. Shyu Y-IL, Liang J, Wu C-C, Cheng H-S, Chen M-C. An interdisciplinary intervention for older Taiwanese patients after surgery for hip fracture improves health-related quality of life. BMC Musculoskelet. Disord. 2010;11(1):225.

20. Cifu DX, Lew HL, Oh-Park M. Geriatric rehabilitation: Elsevier Health Sciences; 2018.

21. Riemen $\mathrm{AH}$, Hutchison JD. The multidisciplinary management of hip fractures in older patients. J. Orthop. Trauma. 2016;30(2):117-22.

22. Shyu YIL, Liang J, Wu CC, Su JY, Cheng HS, Chou SW, et al. Two-year effects of interdisciplinary intervention for hip fracture in older Taiwanese. J. Am. Geriatr. Soc. 2010;58(6):1081-9.

23. Feng $L$, Scherer SC, Tan BY, Chan G, Fong NP, Ng TP. Comorbid cognitive impairment and depression is a significant predictor of poor outcomes in hip fracture rehabilitation. Int. Psychogeriatr. 2010;22(2):246.

Disclaimer: None to declare.

Conflict of Interest: None to declare.

Funding Sources: None to declare. 